\title{
THE OPTIMAL MODEL OF A PROGRAM WITH NEGATION
}

\author{
J.P. DELAHAYE \\ V. THIBAU
}

\author{
L.I.F.L. U.A.-C.N.R.S. n³ $^{\circ} 69$ \\ BAT. M3 \\ Université des Sciences et Techniques de Lille Flandres Artois \\ 59655 Villeneuve d'Ascq Cedex \\ France
}

\begin{abstract}
Abstact: The aim of this paper is to provide another denotational semantics than the least fixpoint semantics for a logic program with negation.

It takes place in a more general work about three-valued (partial) logic and logic programming. We have developped a logical and algebrical theory which seems wellsuited to logic programs with negation. In this theory, we have extended the "consequence" operator of Van Emden and Kowalski associated with a program without negation to a "consequence" operator for programs with negation taking inconsistent programs in account. As the models of a program are exactly the post-fixpoints of this operator, the first provided denotational semantics of a program is the least fixpoint semantics.
\end{abstract}

As an intersection of three-valued Herbrand models of a program is still a model of this program, this least fixpoint is also the set of all the ground literals of a program which are three-valued logical consequences of this program. Even if the least model is thus unique and defined for every consistent program, it is a partial model and may contain only few literals. A second denotational semantics is given by considering the three-valued Herbrand interpretations as partial functions from the set of ground atoms to the set of the truth values $\{T, F\}$; the theory of Manna and Shamir can be adapted in two ways:

- The first one is the notion of optimal fixpoint of the operator.

- The second one seems more interesting for our purpose, since the models of a logic program with negation are exactly the post-fixpoints of the consequence operator. It is the notion of optimal post-fixpoint of our "consequence" operator, which is the optimal model of our program. This notion is about to strengthen the three-valued logic. This optimal model is still unique and defined for every consistent program. It is still a partial model but generally strictly contains the least model, and thus really provides another denotational semantics than the least fixpoint semantics for a program with 
negation. It may even be more interesting since it contains more informations on the program itself.

\section{Introduction}

Situation of the work.

It is well-known that the usual semantics for logic programs without negation: least model, least fixpoint of the immediate "consequence" operator cannot be used for logic programs with negation. The ways of studying negation in logic programming can be divided in two ways (which can interfere). The first one is to consider partial models, for example the well-founded semantics of Van Gelder [26], the weak well-founded semantics of Fitting [11]. In this approach, negation is not allowed in the head of the rules. The weak well-founded semantics of Fitting is the least model of the completed program and the least fixpint of its operator. In the completed program, negation plays a particular part since $\neg p$ is added to the program where $p$ is a predicate symbol appearing in the program without being in the head of a rule. The second way is to consider a partial kind of programs where the use of negation is restricted. For example, stratifiable programs studied by Apt, Blair and Walker [1], have a minimal supported model. Less strong constraints than stratification were also introduced: local stratification by Przymusinski [22], effective stratification by Bidoit and Froidevaux [4c]. For a good survey on negation in logic programming, the reader is sent to [4d].

We have chosen to use partial logic because it generalizes all the results obtained for logic programs without negation: intersection of models property, least model, least fixpoint of the immediate "consequence" operator, and finally the completness of the SLDNF resolution which was proved by Kunen [13b]. This logic gives a symmetry to the negation by introducing a third truth value Indefinite with the order $I \leq F, I \leq T$; the truth values True and False are on the same level and the Indefinite truth value plays the part played by False in classical logic. We have tried to preserve this symmetry without treating negation in a particular way: we do not use negation as failure, we give the semantics of the program itself and not the semantics of its completion even if we can get Fitting's results in our theory and we allow the head of the rules to be negative literals.

We first give the least three-valued model's semantics which seems very natural since it computes all the ground literals which are logical consequences of the program and is also computed by "forward chaining". The logic being partial, this semantics can seem too weak since there are only few logical consequences. The aim of this paper is to find an answer to this weakness by giving another semantics: the optimal model.

\section{Presentation of the paper.}

The first section of this paper is devoted to introduce the theory in which the work takes place. Our theory was conceived to give an answer to the problem of negation in logic programming. This answer, although not being faithful to the negation as failure, provides a good logical and algebrical theory for programs with negation.

This theory has similar properties to those obtained for logic programming without negation. It may be considered, even in its conception as an extension of the theory used for logic programs without negation. This parallel lies in the introduction of a third truth value Indefinite and another implication connective denoted by $\rightarrow$. This connective is neither Lukasiewicz' one as in Lassez and Maher [14] nor the usual Kleene's one. The formula $P \rightarrow Q$ thus has another expressivity than the formula $\neg P \vee Q$. With this third truth value Indefinite and this implication connective, we obtain the intersection models property.

The set of Herbrand interpretations associated with the third value Indefinite is denoted by IHT(L). The members of the set IHT(L) are partial functions from the set of the ground atoms to the set $\{T, F\}$, not being defined where they take the Indefinite value. In 
the first section, we try to present our formalism both as an extension of the case of programs without negation and as an adaptation of Manna and Shamir's formalism.

In the second section, we have also extended the "consequence" operator of Van Emden and Kowalski [25] to a "consequence" operator associated with programs with negation, taking in account the inconsistent programs. The models of a program are exactly the post-fixpoints of this operator. We thus provide the first denotational semantics of a consistant program. It is the least fixpoint of the "consequence" operator. Since the least fixpoint and the least post-fixpoint of a monotonic operator are the same, the least threevalued model of a consistent program is exactly the least fixpoint of the "consequence" operator associated with it. It is also the set of all the ground literals which are logical consequences of a program.

This least model is unique and defined for every consistent program: there is no restriction on the logic studied programs like stratification [4]. Nevertheless, it is a partial model and may contain too many few logical consequences. Another denotational semantics is provided by the structure of the set of three-valued Herbrand interpretations (Fitting [9], Manna et Shamir [16], [17]). The union of two Herbrand three-valued interpretations is not a three-valued Herbrand interpretation except when it is a consistent union. Thus the set of three-valued Herbrand interpretations, denoted by IHT(L), is not a complete lattice as in the bivalued case but only a semi-complete one:

- every nonempty subset of IHT(L) has a glb (greatest lower bound)

- every consistent subset has a lub (lowest upper bound)

The members of the set IHT(L) are partial functions from the set of the ground atoms to the set $[T, F]$, not being defined where they take the Indefinite value. The "consequence" operator is a monotonic functional defined on the set of partial functions from the set of ground atoms to the set (True, False). The theory of Manna and Shamir [16], [17], may be adapted in two ways and provides two other semantics for a logic program with negation.

In the third section, we present the first one: the optimal fixpoint of our "consequence" operator, which is a monotonic functional defined on the set of partial functions from the set of ground atoms to the set (True, False). It is defined as the greatest consistent operator's fixpoint and coincides with the greatest fixpoint less than or equal to the intersection of all the maximal fixpoints.

The fourth and last section is devoted to the second one which is easier and better-suited to our theory. It is the optimal model of a program or the optimal post-fixpoint of the operator. It can be defined as the intersection of all the maximal post-fixpoints which is a post-fixpoint and thus a model, while the intersection of all the maximal fixpoints is not necessarly a fixpoint. We then show that it coincides with the greatest consistent model.

We will see with some examples, that the least model and the optimal one are distinct while they coincide in Lassez and Maher's theory [14]. This gives a special interest to this notion which generally stictly contains the least model.

\section{Partial functions and partial logic}

We try to present our formalism both as an extension of the case of programs without negation and as an adaptation of Manna and Shamir's [16], [17] formalism.

The notion of partial functions (taking the $\omega$ value where not being defined) gives place to partial logic. In a three-valued Herbrand interpretation of our logic, the ground atoms of the language do not necessarly get a truth value belonging to (True, False). In that case, they can get the truth value Indefinite. The following definition gives the notion of three-valued Herbrand interpretation. 
We denote the set of all the ground atoms by her( $(L)$ and the set of all the ground literals by $\operatorname{Her}(\mathrm{L})$.

Definition 1.1 - A three-valued interpretation is a subset $i$ of the set Her(L) such that:

$\forall$ ato $\in$ her(L), ato $\in \mathrm{i}$ implies that $\neg$ ato $\notin \mathrm{i}$

The set of all the three-valued Herbrand interpretations is denoted by IHT(L).

Remarks: 1) We may consider a three-valued Herbrand interpretation as a partial function from her(L) to $[T, F]$. If tvi(ato) is the truth value of an atom ato w.r.t. an interpretation $i$, we can assume that if ato $\notin i$ and $\neg$ ato $\notin i$, then ato does not get a truth value belonging to $(T, F)$ but $t_{i}($ ato $)=I$. If ato $\in i$ then tvi(ato $)=$ True or if $\neg$ ato $\in i$, then tvi(ato $)=$ False.

There is thus a bijection $\phi$ between the set $\operatorname{IHT}(\mathrm{L})$ and the set of the partial functions from her $(L)$ to $[T, F\}$, denoted by $\operatorname{PF}($ her $(L),\{T, F\})$. The mapping $\phi$ is naturally defined by $\phi(i)=t v i$.

2) As in Manna and Shamir [16], [17], the order on the set $\{T, F, I\}$ is the order induced by $I \leq T, I \leq F$. The set $[T, F, I]$ may also be denoted by $[T, F]^{+}$. The corresponding order on IHT(L), which is the order making $\phi$ monotonic, is the set inclusion order on $\operatorname{IHT}(L)$, denoted by $\subseteq$ or $\leq$ as well.

3) The usual notion of Herbrand interpretation, used for programs without negation, which is a member of the set $2^{\text {her }(L)}$, may be considered as a particular case of a threevalued one in the following way:

- A bivalued Herbrand interpretation is a three-valued one such that:

$\forall$ ato $\in$ her(L), ato $\in$ i or $\neg$ ato $\in$ i

The set of all the bivalued Herbrand interpretations is denoted by IHB(L).

To come back to the usual definition, we use the mapping pos: $\operatorname{IHB}(\mathrm{L}) \rightarrow 2^{\text {her }(L)}, \mathrm{i} \rightarrow \mathrm{i}$ $\cap$ her $(L)$, which associates its positive part with an Herbrand bivalued interpretation.

The corresponding order on $\operatorname{IHB}(L)$ is $i \leq j$ iff pos(i) $\subseteq$ pos $(j)$.

4) The partial function tvi: $\operatorname{her}(L) \rightarrow\{T, F\}$, may be extended to a partial function from For( $(L)$ to $[T, F\}$, where For $(L)$ denotes the formula of a logical language $L$ containing the connectives $\wedge, \vee, \neg, \Rightarrow, \Leftrightarrow$, and the quantifiers $\forall, \exists$, by giving the truth value of the connectives $\wedge, \vee, \neg, \Rightarrow, \Leftrightarrow$, and the quantifiers $\forall, \exists$, taking the truth value Indefinite in account.

Definition 1.2 1) We define the truth values of $\wedge, \vee, \neg, \Rightarrow, \Leftrightarrow$ (considered as applications from $(T, F, I)^{2}$ (or $(T, F, I)$ ) to $(T, F, I)$ ).

The truth tables of $\wedge$ and $\vee$ are the following: 


\begin{tabular}{|c|c|c|c|}
\hline$V$ & $T$ & $F$ & $I$ \\
\hline$T$ & $T$ & $T$ & $T$ \\
\hline$F$ & $T$ & $F$ & $I$ \\
\hline$I$ & $T$ & $I$ & $I$ \\
\hline
\end{tabular}

\begin{tabular}{|c|c|c|c|}
\hline$\wedge$ & $T$ & $F$ & $I$ \\
\hline$T$ & $T$ & $F$ & $I$ \\
\hline$F$ & $F$ & $F$ & $F$ \\
\hline$I$ & $I$ & $F$ & $I$ \\
\hline
\end{tabular}

The truth value of $\neg$ is defined by $\neg T=F, \neg F=T, \neg I=I$, the truth value of $P \Rightarrow Q$ is defined as usual by the truth value of $\neg P \vee Q$ and the truth value of $P \Leftrightarrow Q$ by the truth value of $(P \Rightarrow Q) \wedge(Q \Rightarrow P)$.

2) a) We then define the truth value tvi w.r.t. a three-valued i Herbrand interpretation of a closed formula f using the connectives $\wedge, \vee, \neg, \Rightarrow, \Leftrightarrow$, and the quantifiers $\forall, \exists$, inductively by the following:

- If $f=$ ato $\in$ her $(L), t v i(f)=T$ iff ato $\in \mathrm{i}, F$ iff $\neg$ ato $\in \mathrm{i}$, Indefinite otherwise.

- If $f=(t=u)$, (where $t$ and $u$ are two ground terms of the language) $t v i(f)=T$ iff $t$ and $u$ are syntactically identical, False otherwise.

- If $f=\neg g$, or $(g \gamma h)$ where $\gamma \in(\wedge, \vee, \Rightarrow, \Leftrightarrow\}$, the truth value of $f$ is defined as usual with the truth tables above.

- If $f=\forall x g_{*} t v_{i}(f)=T$ iff for every t element of $U n i(L)$ we have $t v_{i}(g(t))=T, F$ iff there is a t element of Uni(L) such that $t v_{i}(g(t))=F$, Indefinite otherwise.

- The same if $f=\exists x \mathrm{~g}$.

b) If $f$ is not closed, $t v_{i}(f)=t v i(\forall f)$ where $\forall f$ is the universal closure of $f$.

This enables us to extend the monotony property obtained in classical logic for the set $\mathrm{F}(\mathrm{L})$ of the formulas making the body of the rules of a program without negation (using the symbols $\wedge, \vee, \forall, \exists)$ to the set For $(L)$ of the formulas making the body of the rules of a three-valued program (a program having rules like lit $\leftarrow$ for, where lit is a literal and the formula for uses the symbols $\wedge, \vee, \neg, \Rightarrow, \Leftrightarrow, \forall, \exists$ ).

In classical logic, the mapping $\psi: 2^{\text {her }(L)} \rightarrow$ (Functions from $F(L)$ to $\left.\{T, F\}\right)$, such that $\psi(i)=t_{i}$ is monotonic. If For $(\mathrm{L})$ is the set of the language's formulas using the symbols $\wedge, \vee, \neg, \Rightarrow, \Leftrightarrow, \forall, \exists$, we have the following proposition:

Proposition 1.1 The mapping $\psi: I H T(L) \rightarrow$ (Partial Functions from For $(L)$ to $\{T$, $F\}$ \}, such that $\phi(i)=v_{i}$ is monotonic for the set inclusion order on $I H T(L)$ and the order induced by $I \leq T, I \leq F$ on the set of the partial functions from $F o r(L)$ to $[T, F\}$.

Proof We have to show that if the formula for uses the symbols $\wedge, \vee, \neg, \Rightarrow, \Leftrightarrow, \forall, \exists$, then $i \subseteq j$ implies that $t_{i}$ (for) $\leq t v j$ (for). By induction on for.

Remarks: - In Przysmusinski [21], we do not have this monotony property, since the order on $\{T, F, I\}$ is the order induced by $F \leq I \leq T$.

- This monotony property will enable us to deduce some important properties about the programs whose bodies' rules belong to For(L). But we must first give another expressivity to the implication connective. 
- If we keep the connective $\Rightarrow$, in the formulas lit $\Leftarrow$ for of a program with negation, we do not yet have the intersection model property

We are now going to extend the logical language $L$ by adding a new implication connective to it. It is neither Lukasiewicz nor Kleene's one, but is denoted by $\rightarrow$, in order to give another expressivity to the implication connective.

Definition 1.3 If $f$ and $g$ are two ground formulas, for any Herbrand interpretation $i$,

$$
\begin{aligned}
& \operatorname{tvi}(f \rightarrow g)=F \text { iff } \operatorname{tvi}(f)=T \text { and } \operatorname{tvi}_{i}(g) \neq T \\
& \text { and } \operatorname{tv}_{i}(f \rightarrow g)=T \text { otherwise. }
\end{aligned}
$$

Remarks: - This connective was introduced in Delahaye [7] and in Shepherdson [23].

- This connective is called non monotonic:

a) Considered as an application from $(T, F, I)^{2}$ to $\{T, F, I]$, with the order induced by $I$ $\leq F, I \leq T$, on these sets, we do not have $\rightarrow(I, F) \leq \rightarrow(T, F)$ although $(I, F) \leq(T, F)$, since $\rightarrow(\mathrm{I}, \mathrm{F})=\mathrm{T}$ while $\rightarrow(\mathrm{T}, \mathrm{F})=\mathrm{F}$.

b) If we include in For(L), the formulas using the connective $\rightarrow$, the mapping $\psi:$ IHT(L) $\rightarrow$ (Partial Functions from For $(L)$ to $[T, F]\}$, such that $\psi(i)=t v i$ is no more monotonic.

At this step of the extension of the truth values and the language, we are now able to get the model intersection property for a Pr program having rules like: lit $\leftarrow$ for, if lit is a literal and the formula for uses the symbols $\wedge, \vee, \neg, \Rightarrow, \Leftrightarrow, \forall, \exists$. We first give the definition of a three-valued Herbrand model of a program with negation in the following definition.

Definitions 1.4 - An Herbrand interpretation i of IHT(L) is a model of a set Ax of formulas of a logical language $L$ if for each formula for of $A x$, we have tvi (for) $=$ True.

- If a set Ax has a model, then $\mathrm{Ax}$ is consistent.

- A formula $f$ of the language is a three-valued logical consequence of Ax if every threevalued model of $\mathrm{Ax}$ is a model of $\mathrm{f}$.

We then have the following intersection model property:

Proposition 1.2 An intersection of three-valued Herbrand models of a program with negation is still a three-valued Herbrand model of this program.

Consequently, for a consistent program, its least Herbrand model denoted by $\mathrm{ltm}(\mathrm{Pr})$ is the intersection of all its Herbrand models and the set of all the ground literals which are logical consequences of Pr.

Proof Pr is a set of rules like lit $\leftarrow$ for, with lit being a literal and the formula for using the symbols $\exists, \forall, \wedge, \vee, \Rightarrow, \neg, \Leftrightarrow$.

Let $\left(i_{e}\right)_{e} \in E$, be a family of Herbrand three-valued models of Pr.

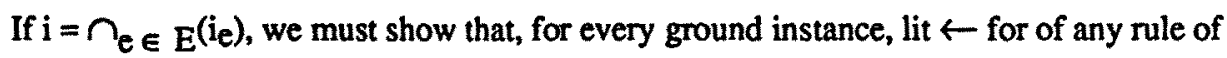
Pr, tvi(lit $\leftarrow$ for $)=$ True.

The only case to check is when tvi(for) $=$ True according to the truth table of $\rightarrow$. $t v i(f o r)=$ True implies that $t_{i} i_{e}$ (for) $=$ True, for each $i_{e}$, by the monotony property, since the formula for uses the symbols $\exists, \forall, \wedge, \vee, \neg, \Rightarrow, \Leftrightarrow$ and $i \subset i_{e}$. As $i_{e}$ is a model of $\operatorname{Pr}, \operatorname{tv}_{\mathrm{e}}\left(\right.$ lit) $=$ True, for each $\mathrm{i}_{\mathrm{e}}$, which means that lit $\in \mathrm{i}_{\mathrm{e}}$ for each $\mathrm{i}_{\mathrm{e}}$, because lit is a literal 
and it follows that lit $\in \mathrm{i}=\cap_{\mathrm{e}} \in \mathrm{E}^{(\mathrm{ie})}$, and so $\mathrm{tvi}_{\mathrm{i}}(\mathrm{lit})=$ True, which implies that tvi(iit $\leftarrow$ for) $=$ True.

So the least Herbrand three-valued model of a consistent program with negation is the intersection of all its Herbrand models.

We now want to characterize this least Herbrand model as a least fixpoint of an operator associated with a program with negation.

2. A monotonic operator on the set of the three-valued interpretations or a monotonic functional on the set of partial functions from her(L) to $\{T, F\}$

Since negation is allowed also in the head of the rules of a program, programs may be not consistent.

To palliate the case of inconsistency of a Pr program, we add an element to the set IHT(L), which we call Contra in the following way:

Definition 2.1 The set IHT' ${ }^{\infty}(\mathrm{L})$ denotes the set IHT(L) $\cup$ [Contra] and the order on $\operatorname{IHT}^{\infty}(\mathrm{L})$ is the order on IHT(L) extended by $\mathrm{i} \subseteq$ Contra for each i element of IHT(L).

We are now able to define the "consequence" operator which is associated with a Pr program with negation.

Definition $2.2 \mathrm{~T}_{\mathrm{Pr}}: \mathrm{IHT}^{\infty}(\mathrm{L}) \rightarrow \mathrm{IHT}^{\infty}(\mathrm{L})$,

$$
\begin{aligned}
& \text { If } i \in \operatorname{IHT}(\mathrm{L}), \mathrm{T}_{\mathrm{Pr}_{\mathrm{r}}}(\mathrm{i})=\{\text { lit } \in \operatorname{Her}(\mathrm{L}) / \text { there is a ground instance lit } \leftarrow \text { for of a rule } \\
& \text { of Pr such that } \left.t v_{i}(\text { for })=\text { True }\right\}
\end{aligned}
$$

if this set does not include an atom and its negation;

$$
\begin{aligned}
& \mathrm{T}_{\mathrm{Pr}}(\mathrm{i})=\text { Contra otherwise; } \\
& \mathrm{T}_{\mathrm{Pr}}(\text { Contra })=\text { Contra. }
\end{aligned}
$$

This operator provides a denotational semantics of a Pr program as it is said in the following theorem.

Theorem 2.1 1) $T_{P r}$ is monotonic and has a least fixpoint denoted by lfp $\left(T_{P r}\right)$.

2) Pr is consistent $\Leftrightarrow l f p\left(T_{P r}\right) \neq$ Contra.

In this case:

a) an interpretation $i \in I H T(L)$ is a model of $\operatorname{Pr} \Leftrightarrow T_{P r}(i) \subseteq i$.

b) $l f p\left(T_{P r}\right)=l \mathrm{tm}(\operatorname{Pr})=\mathrm{C}($ models of $\mathrm{Pr})$.

Proof 1) If $i \in \operatorname{IHT}^{\infty}(\mathrm{L}), \mathrm{i} \subset$ Contra and $\mathrm{T}_{\operatorname{Pr}}(\mathrm{i}) \subseteq$ Contra $=\mathrm{T}_{\operatorname{Pr}}$ (Contra).

Let us have $i$ and $j$ elements of IHT(L).

i $\subseteq j$ implies that $t_{\mathrm{i}}$ (for) $\leq \mathrm{t}_{\mathrm{j}}$ (for) if the formula for only uses the symbols $\exists, \forall, \wedge, \vee$, $\Rightarrow, \Leftrightarrow, \neg$, which is the case for any formula making the body of a rule of Pr.

$\mathrm{T}_{\mathrm{Pr}}$ is thus monotonic. $\mathrm{T}_{\mathrm{Pr}}$ has a least fixpoint because $\mathrm{IHT}^{\infty}(\mathrm{L})$ satisfies the BirkhoffTarski conditions (Lloyd [14]), since the empty set $\varnothing$ is its bottom element and any 
totally ordered set $\left\{I_{\alpha}, \alpha \in A\right\}$ of $\operatorname{IHT}^{\infty}(L)$ has a lub (lowest upper bound) which is Contra if there is $\alpha \in A$ such that $I_{\alpha}=$ Contra and $U_{\alpha \in A^{I}}{ }$, otherwise.

2) To show that $\mathrm{Pr}$ is consistent iff $\operatorname{lfp}\left(\mathrm{T}_{\mathrm{Pr}_{\mathrm{r}}}\right) \neq$ Contra, we first show that:

a) if $i \in \mathbb{I H T}(L)$ is such that $T_{P r}(i) \subseteq i$, then $i$ is a model of $P r$.

Let lit $\leftarrow$ for be a ground instance of a rule of Pr. Let us show that tvi(lit $\leftarrow$ for $)=$ True, if $i \in \mathbb{I H T}(\mathrm{L})$ is such that $T_{\mathrm{Pr}}(\mathrm{i}) \subseteq i$. The only case to check is when $v_{i}($ for $)=$ True.

As $i \in \operatorname{IHT}(L)$ and $\mathrm{T}_{\mathrm{Pr}_{\mathrm{r}}}(\mathrm{i}) \subseteq \mathrm{i}, \mathrm{T}_{\mathrm{Pr}_{\mathrm{r}}}(\mathrm{i}) \neq$ Contra and lit $\in \mathrm{T}_{\mathrm{Pr}_{\mathrm{r}}}(\mathrm{i})$ by the definition of $\mathrm{T}_{\mathrm{Pr}}$ So lit $\in \mathrm{i}$, and $t_{\mathrm{i}}(\mathrm{lit})=$ True.

We then show that:

b) If $i \in \operatorname{IHT}(L)$ is a model of $\operatorname{Pr}$, then $T_{P r}(i) \subseteq i$.

We have tvi(lit $\leftarrow$ for $)=$ True, for every ground instance of a rule of Pr. If lit $\in \mathrm{T}_{\mathrm{Pr}_{\mathrm{r}}(\mathrm{i}) \text {, }}$ then there is a ground instance lit $\leftarrow$ for of a rule of Pr such that $t_{i}($ for $)=$ True. Then $\mathrm{tv}_{\mathbf{i}}(\mathrm{lit})=$ True and $\mathrm{lit} \in \mathbf{i}$.

c) Then we can show by a transfinite induction, that:

If $i \in \mathbb{I H T}(L)$ is a model of $\mathrm{Pr}_{\mathrm{r}}$, then $\mathrm{T}_{\mathrm{Pr}_{\mathrm{r}}} \uparrow \alpha \subseteq i$, for any ordinal $\alpha$.

$\alpha=0, \mathrm{~T}_{\mathrm{Pr}} \uparrow 0=\varnothing$.

If $\alpha$ is a successor ordinal, $\mathrm{T}_{\mathrm{Pr}}\left(\mathrm{T}_{\mathrm{Pr}} \uparrow \alpha-1\right) \subseteq \mathrm{T}_{\mathrm{Pr}}(\mathrm{i})$, by monotony of $\mathrm{T}_{\mathrm{Pr}}$ and induction, and $\mathrm{T}_{\mathrm{Pr}_{\mathrm{T}}}(\mathrm{i}) \subseteq \mathrm{i}$ according to $b$ ) above because $i \in \mathrm{IHT}(\mathrm{L})$ is a model of Pr.

If $\alpha$ is a limit ordinal, $\mathrm{T}_{\mathrm{PT}} \uparrow \alpha=\operatorname{lub}\left\{\mathrm{T}_{\mathrm{Pr}} \uparrow \beta, \beta<\alpha\right\} \subseteq \mathrm{i}$, by induction.

As Ifp $\left(T_{P_{r}}\right)=T_{P_{r}} \uparrow \alpha$, for an ordinal $\alpha$, Ifp $\left(T_{P_{r}}\right) \subseteq i$, for any i model of Pr.

If $\mathrm{Pr}$ is consistent, there is $i \in \mathrm{IHT}(\mathrm{L})$ such that $\mathrm{i}$ is a model of $\mathrm{Pr}$, and $\operatorname{lfp}_{\mathrm{P}}\left(\mathrm{T}_{\mathrm{Pr}}\right) \subseteq \mathrm{i}$ so $\operatorname{lfp}\left(\mathrm{T}_{\mathrm{Pr}}\right) \neq$ Contra.

Conversely, if $\operatorname{lfp}\left(\mathrm{T}_{\mathrm{Pr}_{\mathrm{r}}}\right) \neq$ Contra, $\operatorname{lfp}\left(\mathrm{T}_{\mathrm{Pr}}\right) \in \mathrm{IHT}(\mathrm{L})$ and $\mathrm{T}_{\mathrm{Pr}_{\mathrm{r}}}\left(\operatorname{lfp}\left(\mathrm{T}_{\mathrm{Pr}}\right) \subseteq \operatorname{lfp}\left(\mathrm{T}_{\mathrm{Pr}}\right)\right.$ so Ifp $\left(T_{P_{r}}\right)$ is a model of $\mathrm{Pr}$ which is consistent.

We have shown that $1 \mathrm{fp}\left(\mathrm{T}_{\mathrm{Pr}_{\mathrm{r}}}\right)$ is a model of $\mathrm{Pr}$ when it is $\neq$ Contra.

As we have $\operatorname{lfp}\left(\mathrm{T}_{\mathrm{Pr}}\right) \subseteq \mathrm{i}$, for any $\mathrm{i}$ model of $\mathrm{Pr}$, when $\mathrm{Pr}$ is consistent, $1 \mathrm{fp}\left(\mathrm{T}_{\mathrm{Pr}}\right)=\operatorname{ltm}(\mathrm{Pr})$ $=\cap$ (models of $\mathrm{Pr}$ ), according to the proposition 1.1.

Remarks: 1) In case of a definite program, having rules like lit $\leftarrow$ lit $1, \ldots$, lit $n$, where lit is a literal without the equality predicate and the lit $f_{1}$ are literals, $\mathrm{T}_{\mathrm{Pr}}$ is continuous and $\mathrm{T}_{\mathrm{Pr}} \uparrow \omega=\operatorname{lf} \mathrm{p}\left(\mathrm{T}_{\mathrm{Pr}}\right)$.

2) This operator may be related to Van Emden and Kowalski's one [25] when $\operatorname{Pr}$ is a program without negation, having rules like ato $\leftarrow$ for, where the formula for uses the symbols $\exists, \forall, \wedge, \vee$ with the mapping pos by $\mathrm{B}_{\mathrm{PT}_{\mathrm{T}}}=$ pos $^{-1} \mathrm{oT}_{\mathrm{Pr}_{\mathrm{T}}}$ opos which implies that $\operatorname{lfp}\left(B_{P_{\mathrm{T}}}\right)=\operatorname{pos}^{-1}\left(\mathrm{lfp}\left(\mathrm{T}_{\mathrm{Pr}_{\mathrm{r}}}\right)\right)$ and $\mathrm{lbm}(\mathrm{Pr})=\operatorname{pos}^{-1}(\mathrm{ltm}(\mathrm{Pr}))$ (Delahaye [7], Thibau [24]). It can also be related to Fitting's operator when $\mathrm{Pr}$ is a program with only positive rules like ato $\leftarrow$ for, where the formula for uses the symbols $\wedge, v, \neg, \Rightarrow, \Leftrightarrow, \forall, \exists$ (Fitting [9]). For this relation, we must complete the program (Delahaye [7], Thibau [24]). In our 
theory, the specification is the program itself and our operator provides a denotational semantics of the program itself while the fixpoints of Fitting's operator are the models of the completed program.

3) We have an operational semantics of a program by computing this least three-valued model. With purely syntactical operations on interpretations and programs, every bivalued interpreter may be transformed into a three-valued one (Delahaye[7], Thibau [24]). This has been done for Prolog interpreters (Delahaye-Matthieu [8]) and is actually a classical method used for expert systems working in forward chaining.

\section{Another denotational semantics to palliate the weakness of partial logic.}

This least three-valued model, which gives a first denotational semantics for a program with negation, seems a too weak notion in three-valued logic. With this logic, we do not obtain enough logical consequences. If $\mathrm{Pr}$ is the following program:

$$
\begin{aligned}
& q(x) \leftarrow p(x) \\
& \neg q(a)
\end{aligned}
$$

The least model is $\{\neg q(a)\}$; it does not contain $\neg p$ (a) while it seems natural to have $\neg p(a)$ in the intended model of $\operatorname{Pr}$ since $p(a)$ gives a contradiction by giving both $q(a)$ and $\neg q(a)$ in a model of the program. The weakness of this partial logic is that $\neg p(a)$ is not a logical consequence of $\mathrm{Pr}$ as in bivalued logic. It essentially comes from the fact that the formula $P \rightarrow Q$ is not logically equivalent to $\neg Q \rightarrow \neg P$, while $P \Rightarrow Q$ is logically equivalent to $\neg Q \Rightarrow \neg P$ ).

We want to have an intended model to provide another semantics. According to Kunen [12], the semantics of a logic $P$ program, is not the least fixpoint of the $T$ operator but $T \uparrow \omega$, which is not always a model of P. We prefer to give another model of Pr for another denotational semantics than the least fixpoint.

The first idea, to provide another denotational semantics which contains more informations on a Pr program with negation, was to adapt Manna and Shamir theory for our $\mathrm{T}_{\mathrm{Pr}_{\mathrm{T}}}$ operator. This will be done in the next section. However the optimal fixpoint of $\mathrm{T}_{\mathrm{Pr}}$ will not be chosen for another denotational semantics. We prefer the notion of optimal model which seems better-suited to our programs since their models are not the fixpoints of $\mathrm{T}_{\mathrm{Pr}}$ but its post-fixpoints.

\section{The results of Manna and Shamir for our $T_{\operatorname{Pr}}$ operator [16]}

Since we are interested in a denotational semantics of a consistent program, from now on, we only consider consistent programs.

In the whole section, we assume that the studied three-valued program is consistent.

The considered set is IHT(L); IHT(L) being the set of the Herbrand three-valued interpretations, which are the partial functions from her(L) to $[T, F]$ (taking the truth value Indefinite, where not being defined). This set is not a complete lattice on the contrary of the set of the bivalued interpretations: an union of three-valued Herbrand interpretations is no more a three-valued Herbrand interpretation except if it is a consistent union. 
Definition 3.1 a)Two $i$ and $j$ interpretations of IHT(L) are consistent if $\forall$ ato $\epsilon$ her(L), $i($ ato $) \neq I$ and $j($ ato $) \neq I \Rightarrow i($ ato $)=j$ (ato).

Two elements $i$ and $j$ of IHT(L) are consistent iff the associated partial functions from her(L) to $\{T, F\}, t v_{i}$ and $t v_{j}$ are consistent.

b) A subset $X$ of $\operatorname{IHT}(L)$ is consistent if $\forall i, j \in X, i$ and $j$ are consistent.

Proposition 3.1 a) Every nonempty consistent subset I of IHT(L) has a lub: $\cup_{i \in l} i$.

b) Every nonempty subset I of $I H T(L)$ has a glb: $\bigcap_{i \in f}$.

Proof a) If $I$ is consistent, $\cup_{I \in I} I^{i}$ is a three-valued Herbrand interpretation because this set does not include an atom and its negation.

b) Clear.

\section{Definitions 3.2}

The set of $T_{P r}$ 's fixpoints is $F P\left(T_{P r}\right)=\left\{f \in I H T(L) / f=T_{P r}(f)\right\}$.

The set of $T_{P_{r}}$ 's consistent fixpoints is $C_{F P}\left(T_{P r}\right)=\left[f \in F P\left(T_{P r}\right) / f\right.$ is consistent with any fixpoint of $\mathrm{T}_{\mathrm{Pr}}$.

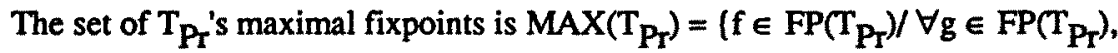

$$
f \leq g \Rightarrow f=g) \text {. }
$$

The set of $T_{P_{r}}$ s pre-fixpoints is $\operatorname{PRE}\left(T_{P_{r}}\right)=\left\{f \in \operatorname{IHT}(L) / f \leq T_{P_{r}}(f)\right\}$.

The set of $T_{\mathrm{Pr}}$ 's post-fixpoints is $\operatorname{POST}\left(\mathrm{T}_{\mathrm{Pr}_{\mathrm{r}}}\right)=\left\{\mathrm{f} \in \mathrm{IHT}(\mathrm{L}) / \mathrm{T}_{\mathrm{Pr}_{\mathrm{r}}}(\mathrm{f}) \leq \mathrm{f}\right\}$.

Lemma 3.1 The program Pr being consistent, all these sets are nonempty.

Proof $\operatorname{Pr}$ is consistent $\Leftrightarrow \operatorname{lfp}\left(\mathrm{T}_{\mathrm{Pr}}\right) \neq$ Contra according to theorem 2.1 , so FP(T $\left.\mathrm{T}_{\mathrm{Pr}}\right)$, CFP( $\left(\mathrm{T}_{\mathrm{Pr}}\right), \operatorname{PRE}\left(\mathrm{T}_{\mathrm{Pr}}\right), \mathrm{POST}\left(\mathrm{T}_{\mathrm{Pr}}\right)$ are nonempty since lfp $\left(\mathrm{T}_{\mathrm{Pr}}\right)$ is a member of all these sets.

As $\operatorname{PRE}\left(\mathrm{T}_{\mathrm{Pr}}\right) \neq \varnothing, \mathrm{MAX}\left(\mathrm{T}_{\mathrm{Pr}}\right) \neq \varnothing$, according to the following lemma:

Lemma 3.2 $\forall f \in \operatorname{PRE}\left(T_{P_{r}}\right), \exists g \in M A X\left(T_{P_{r}}\right)$, such that $f \leq g$.

Proof We first must prove the following lemma:

Lemma 3.3 a) If $f \in P R E\left(T_{P r}\right)$, then the set $\left\{f^{*} \in F P\left(T_{P r}\right), f \leq f\right]$ has a least element. b) Iff $\in P O S T\left(T_{P r}\right)$, the set $\left\{f^{\prime} \in F P\left(T_{P r}\right), f^{\prime} \leq f\right\}$ has a greatest element.

Proposition 3.2 $C F P\left(T_{P_{r}}\right)$ has a greatest element which is the $T_{P r}$ 's optimal fixpoint denoted by opt $\left(T_{P r}\right)$.

Definition 3.3 Since $\mathrm{MAX}\left(\mathrm{T}_{\mathrm{Pr}}\right) \neq \varnothing$, it has a greatest lower bound denoted by $\operatorname{Imax}\left(\mathrm{T}_{\mathrm{Pr}}\right)$. 
Theorem 3.1 a) $l \max \left(T_{P r}\right) \in P O S T\left(T_{P r}\right)$ and $\left\{f \in F P\left(T_{P r}\right), f \leq l m a x\left(T_{P r}\right)\right\}$ has a greatest element.

b) This greatest element is also equal to $\max \left(C F P\left(T_{P r}\right)\right)=o p t\left(T_{P r}\right)$.

For the whole theory of the maximal fixpoint of a monotonic functional on a set of partial functions, and the proofs of these propositions, the reader may read, Manna et Shamir [16], [17].

Remarks: - The optimal fixpoint of $\mathrm{T}_{\mathrm{Pr}}$ may be obtained in two ways, one from the maximal fixpoints, the other one from the consistent fixpoints.

- If $\operatorname{lfp}\left(T_{P r}\right)$ is a total function, i.e. $\neq I$ everywhere, then $\operatorname{lf} p\left(T_{P r}\right)=\operatorname{opt}\left(T_{P r}\right)$, since lfp $\left(T_{P_{r}}\right) \leq \operatorname{opt}\left(T_{P_{r}}\right)$.

- If opt $\left(T_{\mathrm{Pr}}\right)$ is maximal, since $\forall \mathrm{g} \in \operatorname{MAX}\left(\mathrm{T}_{\mathrm{Pr}}\right)$, opt $\left(\mathrm{T}_{\mathrm{Pr}}\right) \leq \operatorname{lmax}\left(\mathrm{T}_{\mathrm{Pr}_{\mathrm{r}}}\right) \leq \mathrm{g}$, we have $\operatorname{opt}\left(\mathrm{T}_{\mathrm{Pr}_{\mathrm{r}}}\right)=\mathrm{g}$ and there is only one maximal fixpoint, $\operatorname{opt}\left(\mathrm{T}_{\mathrm{Pr}}\right)$.

- As Ifp $\left(T_{\mathrm{Pr}_{\mathrm{r}}}\right) \leq \operatorname{opt}\left(\mathrm{T}_{\mathrm{Pr}_{\mathrm{r}}}\right)$, the optimal fixpoint of $\mathrm{T}_{\mathrm{Pr}}$ may be more interesting than the least one since it contains more informations about a program, if these two notions do not coincide.

In our theory, they do not generally coincide as it is shown in the following example.

The optimal fixpoint of $\mathrm{T}_{\mathrm{Pr}}$ is not the same as its least fixpoint for the following $\mathrm{Pr}$ program:

$$
\begin{aligned}
& p(x) \leftarrow \neg q(x) \\
& p(a) \leftarrow q(x) \\
& q(x) \leftarrow \neg p(x) \\
& \neg q(a) \leftarrow \neg p(a) \\
& p(x) \leftarrow p(x)
\end{aligned}
$$

The models' lattice of $\mathrm{Pr}$ is the following:

$$
\{p(a), q(a)\} \quad\{p(a), \neg q(a)\}
$$

$\{p(a)\}$

$\varnothing$

We have: $\operatorname{MOD}(\operatorname{Pr})=\{\varnothing,(p(a)\},\{p(a), q(a)\},\{p(a),-q(a)\})$.

$\operatorname{FP}\left(T_{P_{r}}\right)=\{\varnothing,(p(a))\}$.

$\operatorname{CFP}\left(\mathrm{T}_{\mathrm{Pr}}\right)=\{\varnothing,\{\mathrm{p}(\mathrm{a})\}]$.

$\operatorname{opt}\left(\mathrm{T}_{\mathrm{Pr}_{\mathrm{P}}}\right)=\{\mathrm{p}(\mathrm{a})\} \neq 1 \mathrm{fp}\left(\mathrm{T}_{\mathrm{Pr}_{\mathrm{r}}}\right)=\varnothing$.

While for Lassez and Maher's operator [14], these two notions coincide. This especially comes from the fact that, the interpretation constantly equal to True, is always a model of 
a program with Horn clauses. It follows that neither the least model nor the opional model of a program (which are both included in the maximal interpretation constantly equal to True), take the False truth value. The negative informations, which result from the completed program, are not really represented in this semantics model. So this semantics does not seem well-suited to logic programming with negation.

However, for the following Pr program:

$$
\begin{aligned}
& q(x) \leftarrow p(x) \\
& -q(a)
\end{aligned}
$$

The optimal fixpoint is $[\neg q(a)\}$; it still does not contain $\neg p(a)$ while it seems natural to have $\neg p(a)$ in the intended model of Pr. This is why we try to find another denotational semantics which is is an extension of the Manna and Shamir's theory.

As we have seen that $f$ is a model of $\operatorname{Pr} \Leftrightarrow f \in I H T(L)$ and $T_{\operatorname{Pr}}(f) \leq f$, we are now about to adapt the previous theory to the post-fixpoint of a monotonic operator defined on a set of partial functions. This will be even easier since an intersection of models of Pr (of post-fixpoints of $T_{P_{r}}$ ) is still a model of $P_{r}$ while an intersection of fixpoints of $T_{P_{r}}$ is not necessarly a fixpoint of $\mathrm{T}_{\mathrm{Pr}}$

Making the analogy with the theory of Manna and Shamir [16], we now may define the notions of consistent, maximal and optimal models.

\section{An extension of these results to the models of a program with negation \\ Another denotational semantics than the least model: the optimal model}

\section{Definitions 4.1}

The set of the Pr's models is MOD(Pr) $=\left\{f \in \operatorname{IHT}(L) / T_{P_{r}}(f) \leq f\right\}=P O S T\left(T_{P r}\right)$.

The set of the Pr's consistent models is $\mathrm{CMOD}(\mathrm{Pr})=\left(\mathrm{f} \in \mathrm{POST}\left(\mathrm{T}_{\mathrm{Pr}}\right) / \mathrm{f}\right.$ is consistent with every post-fixpoint of $\mathrm{T}_{\mathrm{Pr}}$ ).

The set of the Pr's maximal models is MAXMOD(Pr) $=\left[f \in \operatorname{POST}\left(T_{\mathrm{Pr}}\right) /\right.$

$$
\left.\forall \mathrm{g} \in \operatorname{POST}\left(\mathrm{T}_{\mathrm{Pr}_{\mathrm{r}}}\right), \mathrm{f} \leq \mathrm{g} \Rightarrow \mathrm{f}=\mathrm{g}\right\} \text {. }
$$

Lemma 4.1 MOD $(P r)$ and $C M O D(P r)$ have a smallest element which is $l f p\left(T_{P r}\right)$, and are consequently nonempty

Proof We have shown (theorem 2.1) by a transfinite induction that the least fixpoint of $\mathrm{T}_{\mathrm{Pr}}$ is its least post-fixpoint $\left(\mathrm{T}_{\mathrm{Pr}} \uparrow \alpha \subseteq \mathrm{f}\right.$, if $\mathrm{f}$ is a post-fixpoint of $\mathrm{T}_{\mathrm{Pr}}$, for every $\alpha$ ordinal) and so we have that $\forall g \in \operatorname{POST}\left(\mathrm{T}_{\mathrm{Pr}_{\mathrm{r}}}\right)$, Ifp $\left(\mathrm{T}_{\mathrm{Pr}}\right) \leq \mathrm{g}$, which implies that $\operatorname{lf}\left(\mathrm{T}_{\mathrm{Pr}}\right)$ is consistent with any post-fixpoint.

To show that MAXMOD(Pr) is nonempty, we prove the following proposition.

Proposition 4.1 $\forall M \in M O D(P r), \exists M^{\prime} \in M A X M O D(P r), M \subseteq M^{\prime}$. 
Proof Let $M \in M O D(P r)$ and $A_{M}=\left\{M^{\prime} \in M O D(P r), M \subseteq M^{\prime}\right\}, A_{M} \neq \varnothing$, since $M \in$ AM.

Every totally ordered subset $S$ of $A_{M}$ has an upper bound in $A_{M}, \cup_{M^{*} \in S^{M}}$ :

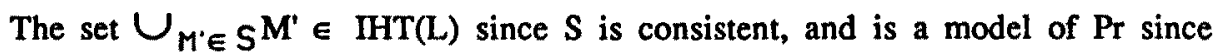

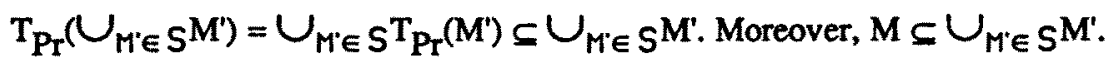

So $A_{M}$ has a maximal element according to Zorn lemma. This maximal element is a maximal model of Pr which contains $\mathrm{M}$.

Proposition 4.2 MAXMOD(Pr) $\neq \varnothing$ and has a greatest lower bound $\operatorname{lmax}(\operatorname{Pr})$ which is a model of $\mathrm{Pr}$.

Proof The program $\operatorname{Pr}$ being consistent, $\mathrm{MOD}(\mathrm{Pr}) \neq \varnothing$ and according to the proposition 4.1, MAXMOD(Pr) $\neq \varnothing$.

As MAXMOD(Pr) $\neq \varnothing, \operatorname{MAXMOD(Pr)~has~a~greatest~lower~bound~}$

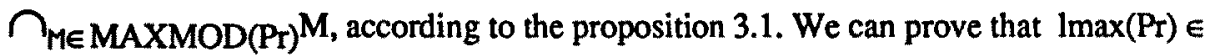
MOD(Pr) in two ways:

- The first one, using the greatest lower bound definition:

$\forall \mathrm{g} \in \mathrm{MAXMOD}(\mathrm{Pr}), \operatorname{lmax}(\mathrm{Pr}) \leq \mathrm{g}$.

So $\forall g \in \operatorname{MAXMOD}(\mathrm{Pr}), \mathrm{T}_{\mathrm{Pr}}(\operatorname{lmax}(\mathrm{Pr})) \leq \mathrm{T}_{\mathrm{Pr}}(\mathrm{g}) \leq \mathrm{g}$, by the monotony of $\mathrm{T}_{\mathrm{Pr}}$ and because $g$ is a post-fixpoint of $\mathrm{T}_{\mathrm{Pr}}$.

So $\mathrm{T}_{\mathrm{Pr}}\left(\max \left(\mathrm{Pr}_{\mathrm{r}}\right)\right) \leq \operatorname{lmax}\left(\mathrm{Pr}_{\mathrm{r}}\right)$, by the glb's definition.

- The second one, using the fact that an intersection of three-valued models of a program is still a model of this program.

Lemma 4.2 and definition 4.2 $C M O D(P r)$ has a greatest element which is $\cup_{M \in C M O D}(\operatorname{Pr})^{M}$ and denoted by opt(Pr).

Proof $\cup_{M \in C M O D(P r)} M \in I H T(L)$ because $C M O D(P r)$ is a consistent set; it is a model of $\operatorname{Pr}$ since $\mathrm{T}_{\mathrm{Pr}}\left(\cup_{M \in C M O D(P r}{ }^{M}\right)=\cup_{M \in \operatorname{CMOD}(\operatorname{Pr})}{ }^{\mathrm{T}}{ }_{\mathrm{Pr}}(\mathrm{M}) \subseteq$ $\left.\cup_{M \in C M O D(P r}\right)^{M}$.

It is consistent:

Let $f$ be a model of $P r$, if $\cup_{M \in C M O D(P r)} M($ lit $) \neq I$ and $f(l i t) \neq I$,

$\exists M_{0} \in C M O D\left(P_{T}\right)$ such that $M_{0}($ lit $) \neq I$ and $M_{0}($ lit $)=U_{M \in C M O D(P r)} M($ lit $)$ by the definition of lub.

As $M_{0}$ is consistent, $M_{0}($ lit $)=f(i t)=\bigcup_{M \in C M O D(P r)} M(l i t)$.

Lemma 4.3 If $g \in M A X M O D(P r)$ and $f \in M O D(P r)$, either $f \subseteq g$, or $f$ and $g$ are not consistent. 
Proof If $f$ and $g$ are consistent, lub(f,g) exists and is equal to $f \cup g$; it is a model of Pr as an union of two consistent models. If not $(f \subseteq g), f \cup g \neq g$ and $g$ would not be a maximal model of Pr.

Theorem 4.1 opt $(P r)=\max (C M O D(P r))=\inf (M A X M O D(P r))=\operatorname{lmax}(P r)$.

Proof We first show that: opt( $(\mathrm{Pr}) \subseteq \operatorname{lmax}(\mathrm{Pr})$;

opt(Pr) is a model of $\mathrm{Pr}$, being consistent with any model of $\mathrm{Pr}$ and according to lemma 4.3, we have: $\forall \mathrm{g} \in \operatorname{MAXMOD}(\operatorname{Pr})$, opt(Pr) $\subseteq \mathrm{g}$, so opt(Pr) $\subseteq \operatorname{lmax}(\mathrm{Pr})$ by the definition of glb(MAXMOD(Pr)).

To show that $\operatorname{lmax}(\mathrm{Pr}) \subseteq \operatorname{opt}(\mathrm{Pr})$, we show that $\operatorname{lmax}(\mathrm{Pr})$ is consistent, which ends the demonstration since $\operatorname{Imax}(\mathrm{Pr})$ is a model of $\mathrm{Pr}$ and $\mathrm{opt}(\mathrm{Pr})$ is the greatest consistent model.

Let $f$ be a model of Pr, then $\exists g \in \operatorname{MAXMOD(Pr),~such~that~} f \subseteq g$, according to the proposition 4.1. As $I \max (\mathrm{Pr}) \subseteq \mathrm{g}$, $\mathrm{f}$ and $\operatorname{lmax}(\mathrm{Pr})$ are consistent.

Remarks 1) The optimal model of a Pr program really defines another denotational semantics than the least model of $\operatorname{Pr}$ (the least fixpoint of $\mathrm{T}_{\mathrm{Pr}}$ ) and the optimal fixpoint of $\mathrm{T}_{\mathrm{Pr}}$ (see ex. below)

2) When the three-valued maximal and the bivalued models are the same, which is the case of pure PROLOG programs, the optimal model is the set of all the ground literals which are the bivalued logical consequences of Pr.

The following programs show both that $\operatorname{opt}(\mathrm{Pr})$ and $\mathrm{Ifp}\left(\mathrm{T}_{\mathrm{Pr}}\right)$ are distinct, and that $\mathrm{opt}(\mathrm{Pr})$ and $\mathrm{opt}\left(\mathrm{T}_{\mathrm{Pr}}\right)$ are distinct.

For the following Pr program:

$$
\begin{aligned}
& q(x) \leftarrow p(x) \\
& \neg q(a)
\end{aligned}
$$

$\operatorname{opt}(\mathrm{Pr})=\{\neg q(\mathrm{a}), \neg p(a))$ while $\operatorname{ltm}(\mathrm{Pr})=\operatorname{lfp}\left(\mathrm{T}_{\mathrm{Pr}}\right)=\{\neg q(\mathrm{a}))$ and $\operatorname{opt}\left(\mathrm{T}_{\mathrm{Pr}_{\mathrm{T}}}\right)=\{\neg q(\mathrm{a}))$.

We finally obtain $\neg p(a)$ in the optimal model which seems natural as we said previously.

The same if $\mathrm{Pr}$ is the following program:

$$
\begin{aligned}
& \mathrm{p}(\mathrm{x}) \leftarrow \neg \mathrm{q}(\mathrm{x}) \\
& \mathrm{p}(\mathrm{a}) \leftarrow \mathrm{q}(\mathrm{x}) \\
& \mathrm{q}(\mathrm{x}) \leftarrow \neg \mathrm{p}(\mathrm{x}) \\
& \neg \mathrm{q}(\mathrm{x}) \leftarrow \neg \mathrm{p}(\mathrm{a})
\end{aligned}
$$

$\operatorname{MOD}(P r)=\{\varnothing,\{p(a)\},\{p(a), q(a)\},(p(a),-q(a))\}$.

$\mathrm{FP}\left(\mathrm{T}_{\mathrm{Pr}_{\mathrm{r}}}\right)=[\varnothing]$.

$\operatorname{MAXMOD}(\operatorname{Pr})=\{(p(a), q(a)\},(p(a), \neg q(a)\})$.

$\operatorname{opt}(\mathrm{Pr})=\{\mathrm{p}(\mathrm{a})\}$ while $\operatorname{ltm}(\mathrm{Pr})=\operatorname{lfp}\left(\mathrm{T}_{\mathrm{Pr}}\right)=\varnothing$ and opt $\left(\mathrm{T}_{\mathrm{Pr}_{\mathrm{r}}}\right)=\varnothing$. 
So the optimal model may define another denotational semantics than the least fixpoint. It gives a concurrent semantics of the intended model of Apt, Blair and Walker [1] for a logic program with negation. Although it is a partial model, it is unique and defined for every consistent program with negation. It is an adaptation of the optimal fixpoint of an operator which has already been introduced in Fitting [9] and in Manna and Shamir [16]. However, in Fitting's theory, the fixpoints of his operator are exactly the models of the completed program. Since in our theory, the specification of a Pr program is the program itself, and its models are the post-fixpoints of the associated "consequence" operator, the notion of optimal model or optimal post-fixpoint seemed a natural extension to us to study logic programs with negation. We will have to study whether it is or not computable in the predicate case and give good algorithms in the propositional case.

\section{REFERENCES}

[1] K. Apt, H.Blair and A.Walker, Towards a theory of declarative knowledge, in: J. Minker, ed, Foundations of Deductive Databases and Logic Programming (Morgan Kaufman, Los Altos, 1988), 89-142.

[2] K. Apt and M. Van Emden, Contribution to the theory of logic programming, J.ACM 29-3 (1982) 841-842.

[3] M. Ben Jacob and M. Fitting, Stratified and Three-valued Logic Programming Semantics, in: R.A. Kowalski and A. Bowen, ed., Proceedings of the Fifth International Conference and symposium on Logic Programming (MIT Press, 1988) $1055-1069$.

[4a] N. Bidoit et C. Froidevaux, Variations sur la contrainte de stratification: Stratification, Stratification locale, Sup-stratification: Journées Bases de données avancees, 1988.

[4b] N. Bidoit et C. Froidevaux, General logic databases and programs: Default logic semantics and stratification,To appear in J. of Information and computation.

[4c] N. Bidoit et C. Froidevaux, Negation by default and Unstratifiable Logic Programs,To appear in TCS.

[4d] N. Bidoit, Negation in Rule-Based Database Language: A Survey, To appear in TCS.

[5] K.L. Clark, Negation as failure, in: Gallaire and Minker ed., Logic and databases (Plenum Press, New York,1978).293-324.

[6] J.P. Delahaye: Chainage avant et calcul booléen et trivalué, Journées internationales sur les systemes experts et leurs applications, Avignon 1987, 1341-1360.

[7] J.P. Delahaye: Programmation en logique trivaluée, rapport de recherche I.T n¹15, Université des Sciences et Techniques de Lille, 1987.

[8] J.P. Delahaye, P. Matthieu: Logique partielle et Prolog, séminaire de programmation logique de Tregastel, 1989.

[9] M. Fitting, A Kripke-Kleene semantic for logic programs, J. Logic Programming 4 (1985) 295-312.

[10] M. Fitting, Notes on the mathematical aspects of Kripke 's theory of truth, Notre Dame Journal of Formal Logic, vol.27, $\mathrm{n}^{\circ} 1$ (1986) 75-88.

[11] M. Fitting, Partial models and logic programming, Theoretical Computer Science 48 (1986) 229-255.

[12] K. Kunen, Negation in Logic Programming, Journal of Logic Programming (1987) 289-308.

[13a] K. Kunen, Some remarks on the completed databases, in: R. Kowalski and K. Bowen, eds, Proceedings of the fifth Logic Programming symposium (Association for Logic Programming, MIT Press, Cambridge, Mass, 1988) 978-992.

[13b] K. Kunen, Signed data dependencies in logic programming, Journal of Logic Programming (1989) 231-245. .

[14] J.L. Lassez et M.J. Maher, Optimal fixpoints of logic programs, Theoretical Computer Science 39 (1985) 15-25. 
[15] J.W. Lloyd, Foundations of Logic Programming (second edition, Springer, Berlin, 1987).

[16] Z. Manna and A. Shamir, The theoretical aspects of the optimal fixpoints, Siam J. comput., vol 5, $n^{\circ} 3$ (1976).414-426.

[17] Z. Manna and A. Shamir, The optimal approach to recursive programs, $A C M$ vol 20, $n^{\circ} 11(1977)$ 824-831

[18] A. Mycroft, Logic programs and many-valued logic,in: STACS 84, Lecture Notes in Computer Science 166 (Springer, Berlin, 1984) 274-286.

[19] T. Przymunsinski and H. Przymunsinski, Weakly perfect model semantics for Logic programs, Conference and Symp. on Logic Programming, (1988) 1106-1120.

[20] T. Przymunsinski, Every Logic Program has a natural Stratification and an iterated fixed point model, Proc. ACM Symp. Principles of Databases Systems, (1988) 1121.

[21] T. Przymunsinski, Non monotonic formalisms and Logic Programming, ICLP, (1989) 655-674.

[22] T. Przymunsinski, On the declarative semantics of deductive databases and logic programs, in: J. Minker, ed, Foundations of Deductive Databases and Logic Programming (Morgan Kaufman, Los Altos, 1988).

[23] J.C. Shepherdson, Negation in Logic Programming, in: J. Minker, ed, Foundations of Deductive Databases and Logic Programming (Morgan Kaufman, Los Altos, 1988) $19-87$.

[24] V. Thibau, Thèse de doctorat: Une logique trivaluée appliqué à la programmation logique, Université des Sciences et Techniques de Lille, 1990.

[25] M.H. Van Emden, R.A. Kowalski, The semantics of predicate logic as a programming language, J. ACM, 23 (1976), 733-742.

[26] A. Van Gelder, The well-founded semantics for general logic programs, Proc. ACM Symp. Principles of Databases Systems, (March 1988) 221-230.

[27] A. Van Gelder, The alternative fixpoint of logic programs with negation, Proc. ACM Symp. Principles of Databases Systems, (March 1989) 1-10. 